\title{
Combination of Metal Shielding and Distance Estimation for Electromagnetic Compatibility Guarantee
}

\author{
Nguyen Duc Truong1, Tran Van Nghia², Bui Duc Chinh ${ }^{3}$, Ho Quang Quy ${ }^{4^{*}}$ \\ ${ }^{1}$ Standard Measurement Quality Department, Hanoi, Vietnam \\ ${ }^{2}$ Faculty of Electronics and Telecommunications, Electric Power University (EPU), Hanoi, Vietnam \\ ${ }^{3}$ Institute of Cryptographic Science and Technology, Hanoi, Vietnam \\ ${ }^{4}$ Ho Chi Minh City University of Food Industry, Ho Chi Minh City, Vietnam \\ Email: ^hoquangquy@gmail.com
}

How to cite this paper: Truong, N.D., Van Nghia, T., Chinh, B.D. and Quy, H.Q. (2019) Combination of Metal Shielding and Distance Estimation for Electromagnetic Compatibility Guarantee. Journal of Electromagnetic Analysis and Applications, 11, 135-147.

https://doi.org/10.4236/jemaa.2019.119009

Received: July 24, 2019

Accepted: September 14, 2019

Published: September 17, 2019

Copyright $\odot 2019$ by author(s) and Scientific Research Publishing Inc. This work is licensed under the Creative Commons Attribution International License (CC BY 4.0).

http://creativecommons.org/licenses/by/4.0/

\begin{abstract}
In this paper, a solution for electromagnetic compatibility guarantee based on the combination of metal shielding and circuit components distance estimation methods is presented. The electromagnetic noises generated from a working radio-electronic unit can expand into the space and act on other around radio-electronic units. An EMC guaranteed radio-electronic unit by the suitable technique method will not cause the electromagnetic noise to others. In opposition, it will not be under electromagnetic action from another one. Due to the power of electromagnetic noise, the metal shielding, distance estimation or other technique methods should be used to guarantee EMC. Every method has own advantage as so as weakness for detail radio-electronic unit, so it is necessary to choose a suitable method to guarantee EMC for them, the combination of metal shielding and distance estimation is a choice, for example. The proposed solution has been evaluated by using CST (Computer Simulation Technology) software and EMxpertEHX analyzer in oscillator circuit context. The simulated results on CST show that the proposed solution decreases the electromagnetic radiation about of $39.1 \mathrm{~dB}$ at frequency $500 \mathrm{MHz}$ in comparison to results when nothing electromagnetic compatibility methods are not used. The experimental results on the oscillator circuit are presented. The electromagnetic radiation reduction of the oscillator circuit is about of ( $25-30) \mathrm{dB}$. In comparison to individual metal shielding and distance estimation methods, the effectiveness of the proposed solution for electromagnetic compatibility guarantee is significantly increased.
\end{abstract}

\section{Keywords}

Electromagnetic Compatibility, Electromagnetic Radiation, Metal Shielding, 


\section{Introduction}

Nowadays, with the huge development of science and technology, the radioelectronic units (REUs) must be in guarantee not of the desired operation efficiency but electromagnetic compatibility (EMC) also before to use (refs. [1] [2] [3] [4] [5]). EMC is the capability to normally operate in the electromagnetic field (EMF) and do not influence the operation of another of the REUs. Some standards of EMC as CISPR-22, EN55022 (in Europe), FCC-15 (in USA), IEC61000 (international) well as TCVN-7189 (in Vietnam) equivalent to CISPR-22 have been popularly using. Although between mentioned standards there is a little of ignorable differences, but all of them are one to other equivalent, so the CISPR-22 will be chosen for this study (ref. [6]). There are a lot of methods used to guarantee EMC for REUs as the electric or electronic circuit design, mechanical processing, print circuit, ... well as the metal shielding (ref. [7] and ref. [8]). Although every method has own advantage and weakness, but for this study, we are only interested in the solution to guarantee EMC of available electronic modules without interfering in the design process of them. A popular technique to guarantee EMC of individual REU or system of modules is the EMF shielding by the metal sheathed box (ref. [9]). By this technique the shielding effectiveness (SE) is very high if the metal sheathed box is designed closed tigth, consequently, the EMF generated from it can be blocked. However, in the real, there are always slits or small holes and apertures for air ventilation or for cable installation in the metal sheath (ref. [10] and ref. [11]). If the slits and holes have not optimally chosen, the SE is significantly reduced. In works (ref. [4] and ref. [5]), authors have simulated and evaluated the influence of slits and holes on the SE and then optimized by choice the suitable collection of their size, shape and distribution in metal sheath. The results in works (ref. [9] and ref. [10]) have shown that it is very difficult and complex to manually control the parameters of holes in the metal sheath for the EMC guarantee. The other method to guarantee EMC is the distance estimation of the electronic components. It is based on the decrease of EMF with propagation distance (ref. [11]). However, there is a weakness of this method that in a distance of some centimeters the decrease of EMF is ignorable small, while in a long distance the decrease of EMF will be significant, but that consequences the REU is bigger and uncomfortable. It is clear that one of two mentioned above methods has own advantages as well as weaknesses to guarantee EMC.

In this paper, we propose a solution to combine both mentioned above methods to guarantee EMC. Before investigation the SE of proposed solution, the metal shielding as well as distance estimation are investigated individually in detail by the CST software (ref. [12]) and EMC guarantee is tested by EMxpertEHX analyzer (ref. [13]). 


\section{Background of Metal Shielding and Distance Estimation for EMC Guarantee}

\subsection{Metal Shielding (MS)}

The main parameter to determine the influence of the metal sheath on EMF is SE related to the ratio of the incident and transmitted electric (magnetic) intensities, which are given as (ref. [11] and ref. [14]):

$$
\begin{gathered}
S E_{e}=20 \lg \left|\frac{E_{i}}{E_{t}}\right| \\
S E_{h}=20 \lg \left|\frac{H_{i}}{H_{t}}\right|
\end{gathered}
$$

where $E_{i}, E_{t}\left(H_{i}, H_{t}\right)$ are the incident and transmitted electric (magnetic) intensities, respectively.

Beyond Equation (1) and Equation (2) given above, as shown in Figure 1 the SE can be formed as the sum of the reflection, $R[\mathrm{~dB}]$, absorption, $A[\mathrm{~dB}]$ and re-reflection (multiple-reflection), $B[\mathrm{~dB}]$ which are given follows:

$$
S E[\mathrm{~dB}]=R[\mathrm{~dB}]+A[\mathrm{~dB}]+B[\mathrm{~dB}]
$$

where,

$$
R_{\mathrm{dB}}=168+10 \log _{10}\left(\sigma_{r} / \mu_{r} f\right)
$$

is the reflection in far field (ref. [14]),

$$
\begin{gathered}
R_{e, \mathrm{~dB}}=322+10 \log _{10}\left(\sigma_{r} / \mu_{r} f^{3} r^{2}\right) \\
R_{m, \mathrm{~dB}}=14.57+10 \log _{10}\left(f r^{2} \sigma_{r} / \mu_{r}\right)
\end{gathered}
$$

are the reflections of electric and magnetic sources in near field, respectively (ref. [14]),

$$
A_{\mathrm{dB}}=20 \log _{10} e^{t / \delta}=20 \frac{t}{\delta} \log _{10} e=1.314 t \sqrt{f \mu_{r} \sigma_{r}}
$$

is the absorption (ref. [14]),

$$
B=20 \lg \left(1-e^{-2 t / \delta}\right),
$$

is the re-reflection (ref. [15]), $\delta=(\sqrt{\pi f \mu \sigma})^{-1}$ is the skin depth of the material. $\mu_{r}$ is the reversible permeability relative to air. $\sigma_{r}$ is the conductivity relative to copper. $\mu$ is the magnetic permeability of material. $\sigma$ is the conductivity

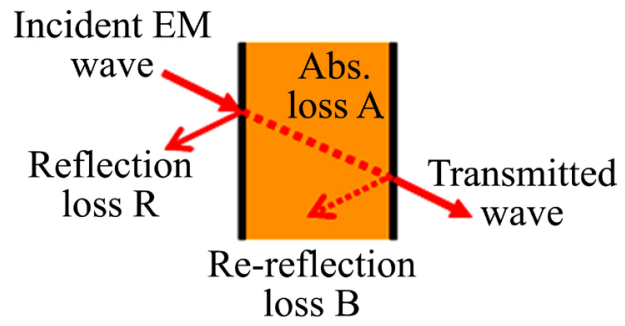

Figure 1. SE description of metal sheath. 
of material. $t[\mathrm{~cm}]$ is the thicknees of material. $r[\mathrm{~cm}]$ is the distance between radiation source and metal sheath. The re-reflection $B$ will be meaningful if $A \leq 15$ [dB] (ref. [14]), only, consequently it is always really ignored, since $A>15[\mathrm{~dB}]$.

\subsection{Distance Estimation between Modules (DE)}

All the results above show that the metal shielding is easy to design, but it needs to eliminate slits and to choose suitable size of holes if they are that depends on technology. So to enhance or perfectly guarantee EMC it is necessary to use distance estimation between RE modules or units combined to metal shielding. As know, the EMF changes with propagation distance, but differently for near and far fields. For the far field, Inverse Distance Method (ref. [15]) is used to determine radiation noice at distance $R$ and $R^{\prime}$ (Figure 2), which is given as follows:

$$
E\left(R^{\prime}\right)[\mathrm{dB}]=E(R)[\mathrm{dB}]+20 \lg \left(R / R^{\prime}\right)
$$

The near field is more complex than far field. The field parts change inversely not linear to distance as $1 / r$, but nonlinear as $1 / r^{2}$ or $1 / r^{3}$ (Figure 3 ).

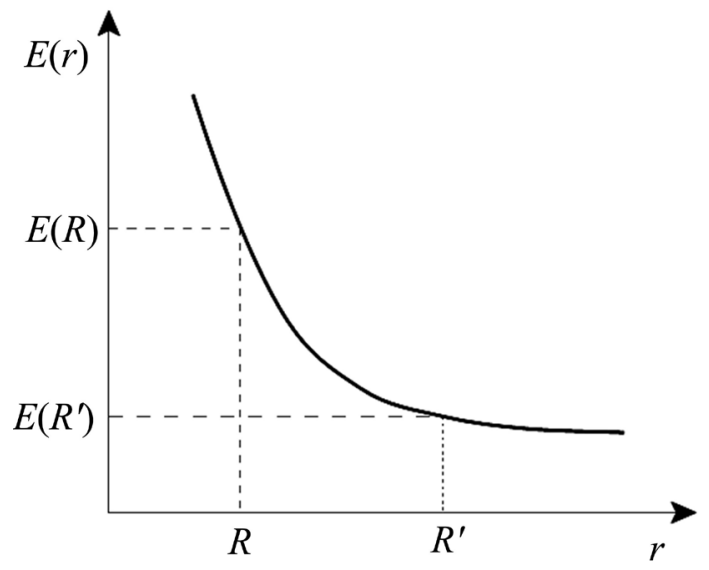

Figure 2. Principle of inverse distance method.

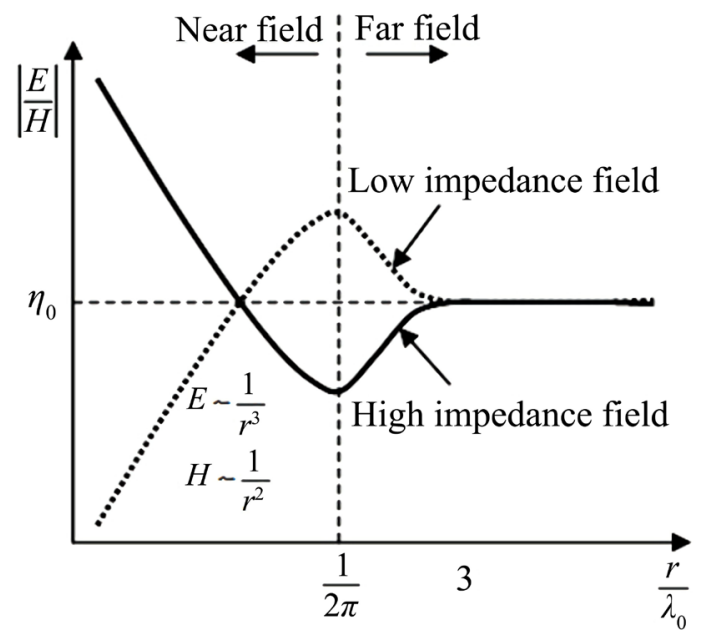

Figure 3. Field parts in near and far fileld. 
The boundary between near and far fields is where the ratio $E / H=Z_{w}$ called wave impedance is approximately equal to internal impedance of free space $\eta_{0}$ (ref. [15]).

\section{Results and Discussions}

\subsection{The Investigation Model}

Consider an aluminium (Al) sheathed box (ASB) with size of $16 \times 10 \times 3 \mathrm{~cm}$ and thickness of sheath of $2 \mathrm{~cm}$ (Figure 4). The coaxial cable with copper core of $0.16 \mathrm{~cm}$ diameter and impedance of $50 \Omega$ is used to put a radiation source placed inside ASB so that the radiation distribution in the upper space around it is homogeneous (ref. [10]).

A second ASB with size of $10 \times 10 \times 3 \mathrm{~cm}$ is placed in distance of $\mathrm{d}$ from the first one. The CST will be used to evaluate SE for different cases as: single ASB, single ASB with holes in the sheath, two ASBs with different distance one from other. The EMC will be simulated and evaluated at frequency changing from 1 $\mathrm{MHz}$ to $1 \mathrm{GHz}$. Beside using CST to evaluate the SE of proposed solution, the oscillation circuits and power supply with output voltage of $5 \mathrm{~V}$ is experimentally evaluated using measurement equipment EMxpertEHX consisting from EMxpert software and EMSCAN scanner (Figure 5).

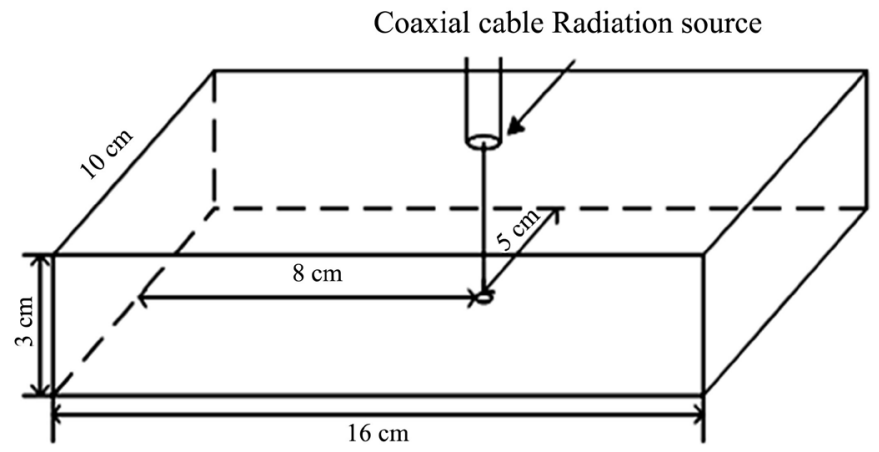

Figure 4. ASB for investigation.

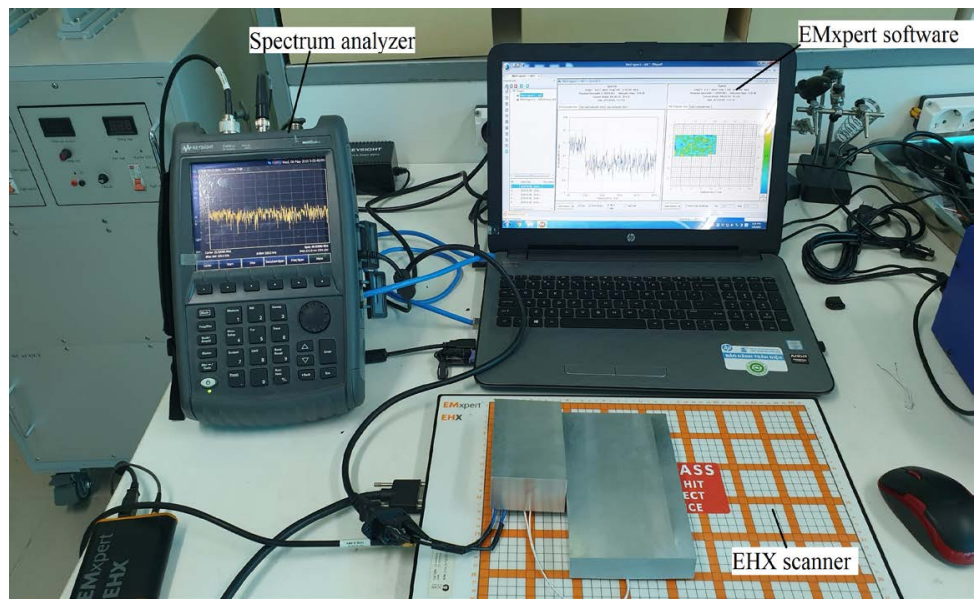

Figure 5. Measurement set-up using EMxpertEHX. 


\subsection{EMC by MS}

Using Equation (3) and aluminium sheath with $t=0.2[\mathrm{~cm}], \mu_{r}=1$ and $\sigma_{r}=0.61$ at frequency from $500 \mathrm{kHz}$ (ref. [15]) the SE-frequency characteristic is calculated and presented in Figure 6. The distribution of electric field inside ASB is simulated and shown in Figure 7.

From Figure 6, we can see that in the frequency band from $30 \mathrm{MHz}$ to $1 \mathrm{GHz}$ of CISPR-22 standard (ref. [6]), the SE speedily increases, event till $1000 \mathrm{~dB}$. It results that any part of electric field (EF) transmitted outside ASB as shown in Figure 7.

However, the ASB is not always closed tight; instead, there are slits or small holes and apertures used for air ventilation or for cable installation, really. If the slits and holes are not suitble designed, the radiation signal leakage may overcome the standard limit as of CISPR-22 then causes inexpectant effects. Figure 8 and Figure 9 are the distribution of the electric field around the ASB with aperture of $1 \times 0.5 \mathrm{~cm}$ size. It is clear that with hole there is the leakage outside of radiation signal (Figure 8). The minimum inside electric intensity is about 100 $\mathrm{dB} \mu \mathrm{V} / \mathrm{m}$ while outside electric intensity is lower than $80 \mathrm{~dB} \mu \mathrm{V} / \mathrm{m}$. Specially, the electric intensity at aperture is equivalent to that inside ASB, meanwhile, it is only about $20-60 \mathrm{~dB} \mu \mathrm{V} / \mathrm{m}$ otherwhere (Figure 9).

Figure 10 presents EMC test is measured at distance $3 \mathrm{~m}$ followed to CISPR-22 standard (ref. [6]). Consider that there is a unshielding signal overcomes limit line of EMC standard (dark line). In the case of closed tight ASB, the EF intensity is very small about $-200 \mathrm{~dB}$ which is the minimum value of simulation.

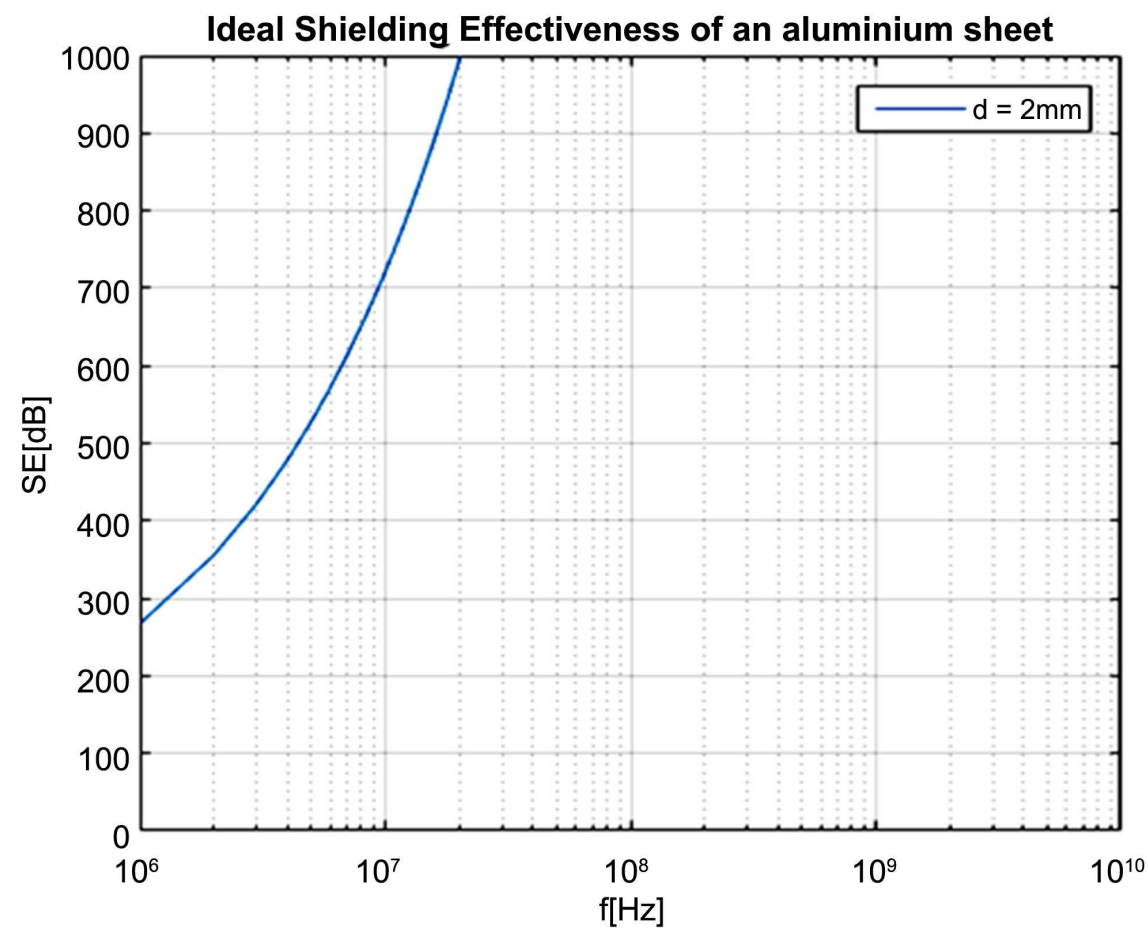

Figure 6. SE-frequency characteristic of Al sheath. 


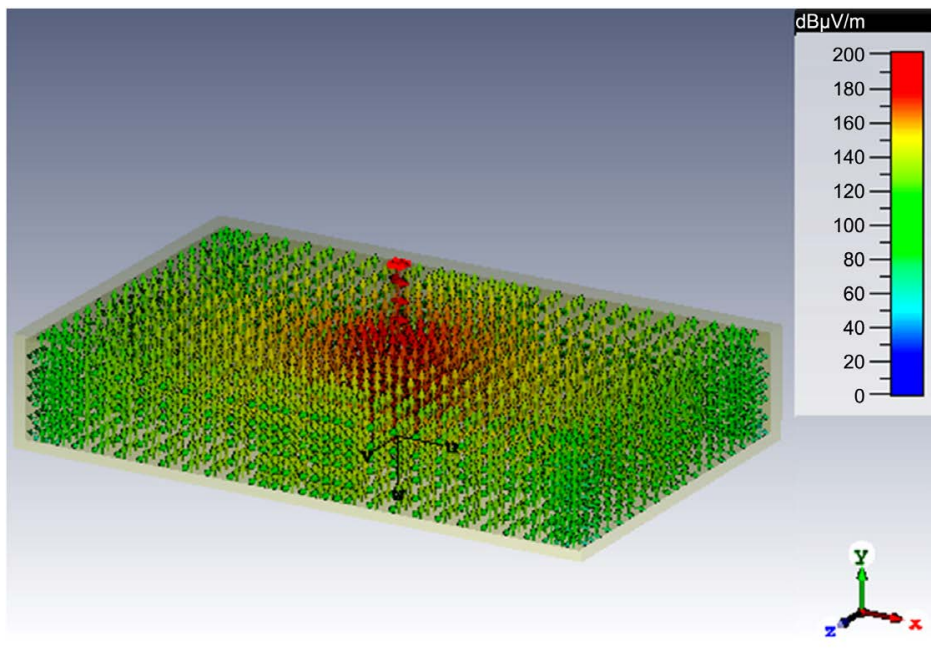

Figure 7. EF distribution in 3D space of ASB.



Figure 8. EF distribution around ASB with hole.

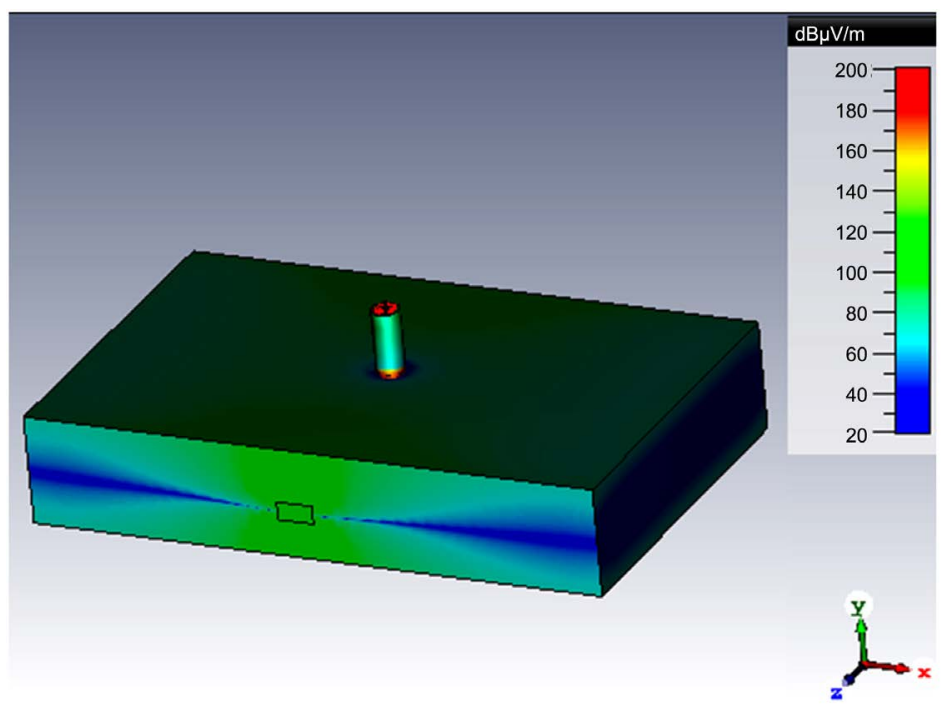

Figure 9. EF distribution on the metal sheath with small aperture. 
EMC: Efield at 3 meter distance $[\mathrm{dB}($ microV/m)]

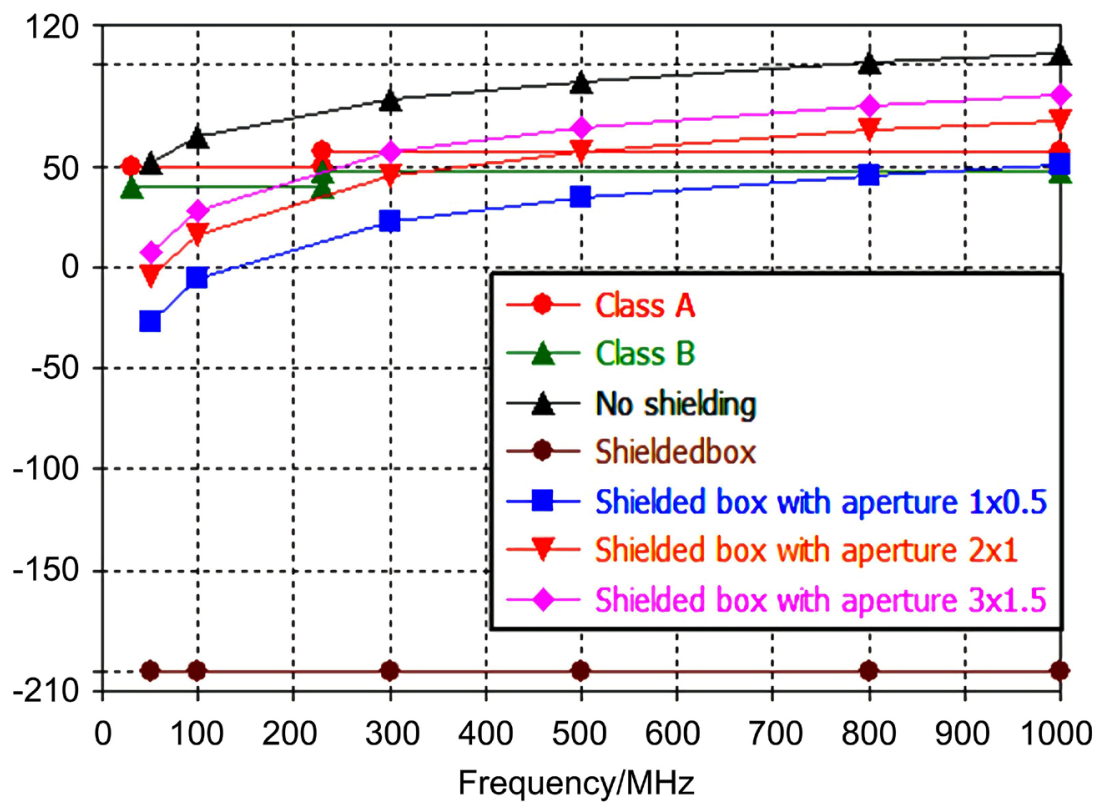

Figure 10 EMC tested $3 \mathrm{~cm}$ for different shielding cases.

For the case of ASB with three apertures of different size of $3 \times 1.5 \mathrm{~cm}$, $2 \times 1.0 \mathrm{~cm}$ and $1 \times 0.5 \mathrm{~cm}$ the EF intensity decreases about $23.1 \mathrm{~dB}, 48.2 \mathrm{~dB}$ and $57.3 \mathrm{~dB}$ in comparison to that of inshielding case measured at $500 \mathrm{MHz}$ frequency. This situation is similar to the case measured at a distance of $2 \mathrm{~cm}$ (Figure 11).

\subsection{MEC by DE}

Using Equation (9) and what described in Figure 2 and Figure 3, the field distribution around the ASB is simulated and presented in Figure 12 and the EF intensity-frequency characteristics at different distances are presented in Figure 13.

From Figure 13, we can see that the electric intensity decreases with the increase of distance till $10 \mathrm{~cm}$ at wide frequency range to $1 \mathrm{GHz}$, but nonlinear if distance is longer than $10 \mathrm{~cm}$. In the distance longer than $10 \mathrm{~cm}$, the electric intensity increases with the increase of frequency. This situation is absolutely similar to that shown in Figure 10 and Figure 11. We can say that the results obtained in Figure 13 are too high, because they are measured in free space. It means the receiver is not EMC guaranteed. The situation will be different for the EMC guaranteed ASB.

\subsection{EMC by Combination of MS and DE}

Let a second ASB with size of $10 \times 10 \times 3 \mathrm{~cm}$ is placed in distance of $\mathrm{d}$ from the first one. The distribution of the EF around both ASBs is simulated and illustrated in Figure 14. It is clear that the EF generated from the radiation source inside the first ASB overlaps a part of the space around the second ASB. Changing 


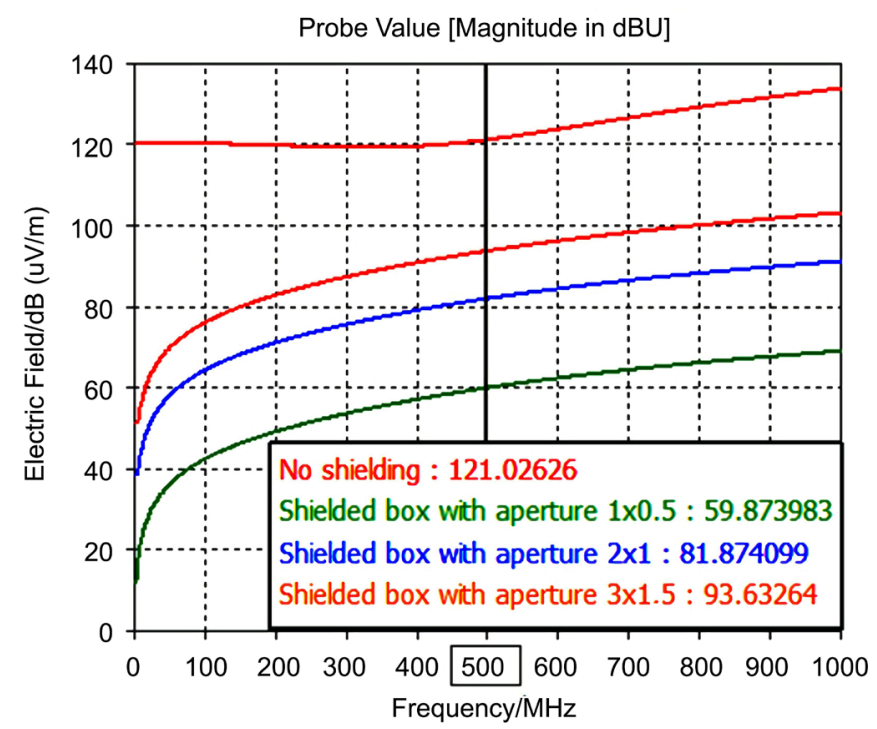

Figure 11. EMC simulated at $2 \mathrm{~cm}$ for different shielding cases.

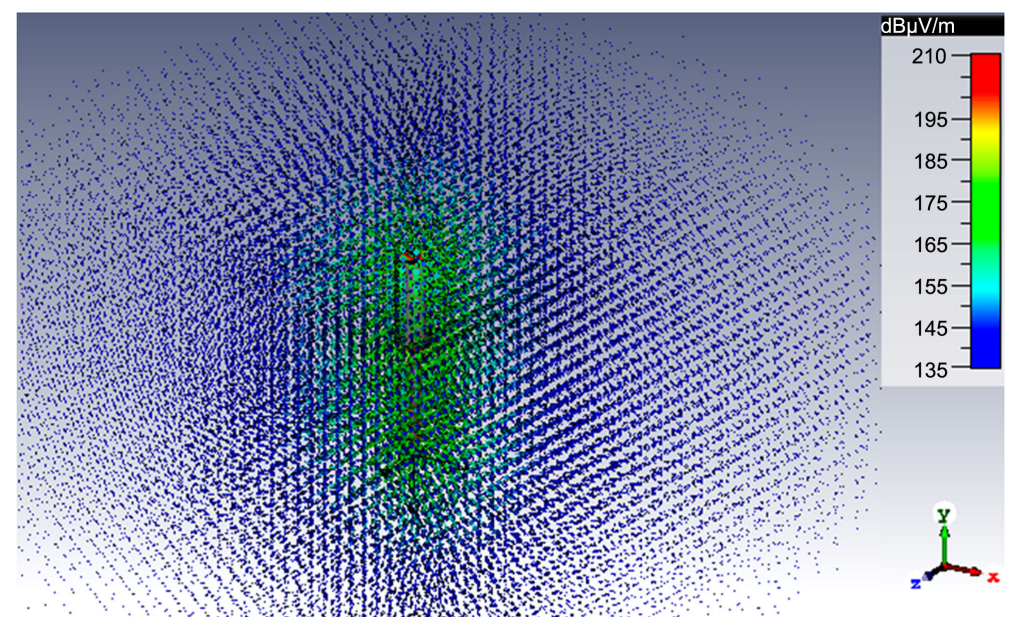

Figure 12. EF distribution around ASB in far field.

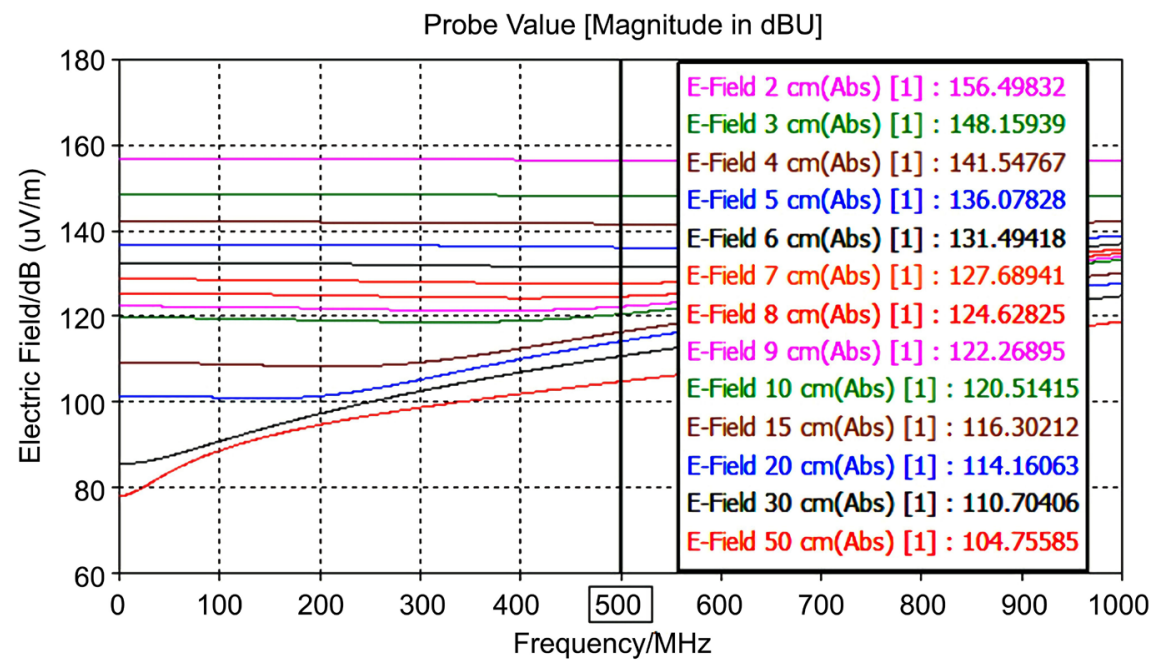

Figure 13. EF intensity-frequency characteristics with different distances. 
the distance between two ASBs, the EF intensity inside second ASB-frequency characteristics with different distances are simulated and illustrated in Figure 15. From Figure 15, we can see that the EF intensity reduces significantly by shielding and linearly decreases with the increase of distance between ASBs, and consequently the metal shielding, as well as the distance estimation, affects directly on the EMC guarantee.

Although EF intensity decreases with the increase of distance, but it does not reject the influence one on other if both of ASBs are in operation regime. This phenomenon is proved when observing the power supply is placed close to the oscillator circuit without shielding (Figure 16).

From the EF distribution in the plane of scanner (Figure 17), we can see the

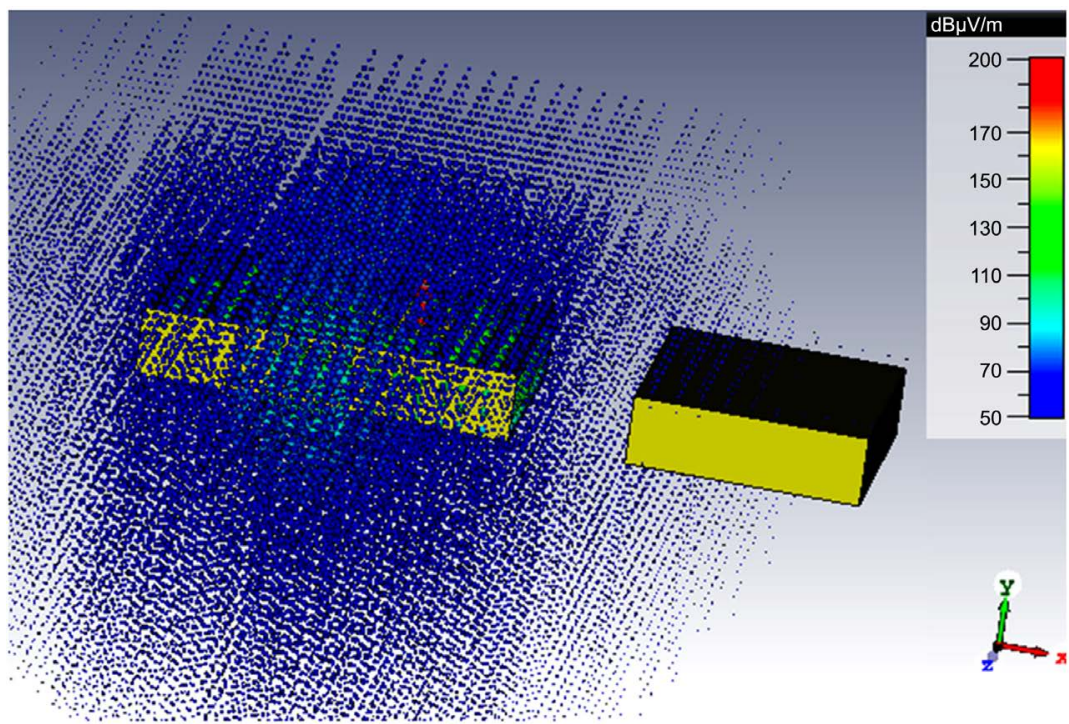

Figure 14. EF distribution around both ASBs.

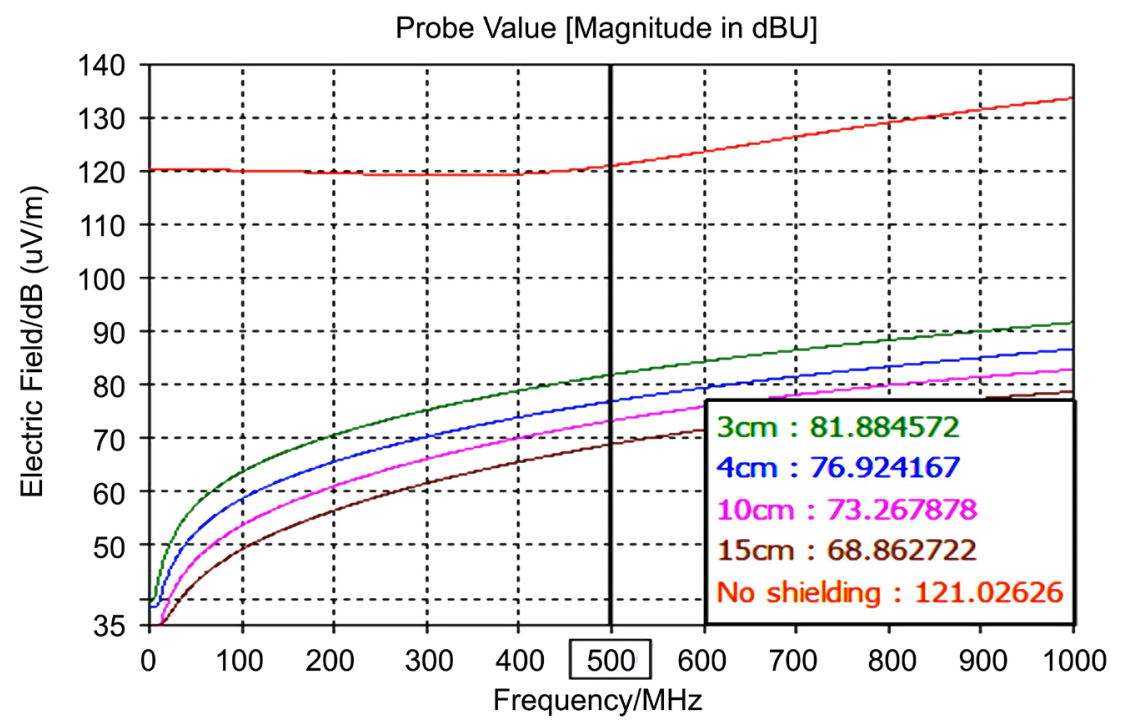

Figure 15. EF intensity inside second ASB-frequency characteristics with different distances between ASBs. 


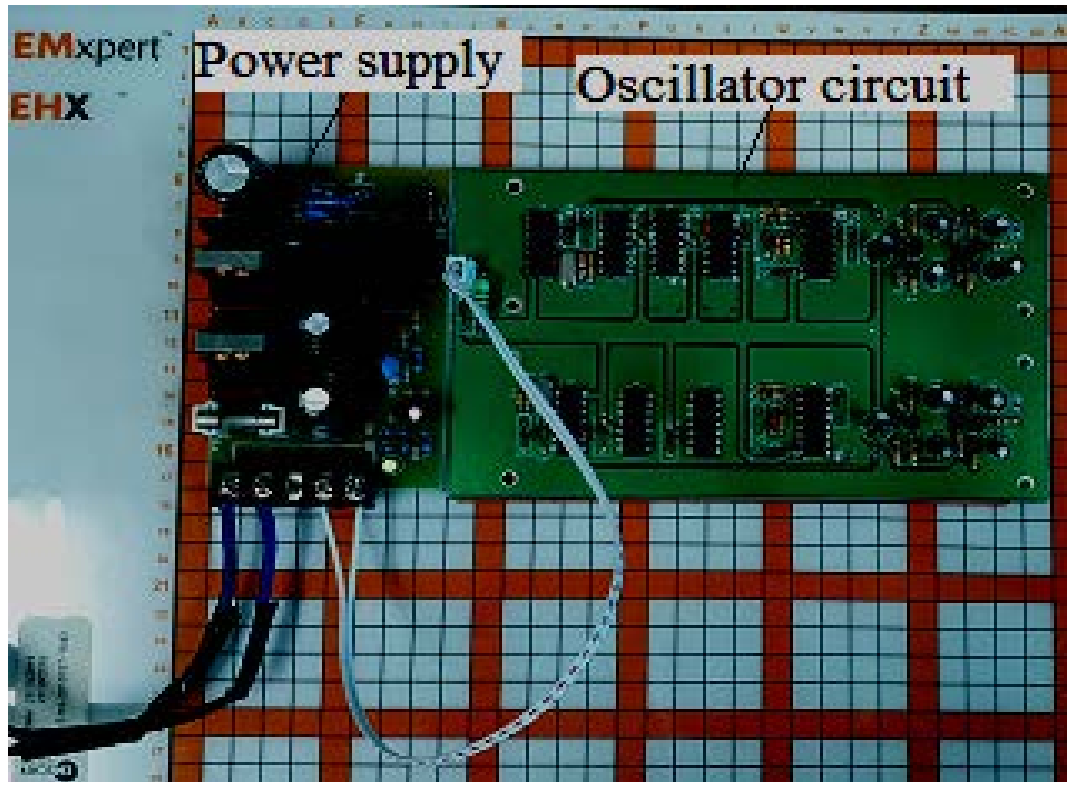

Figure 16. Two inshielded circuits closed one to other.

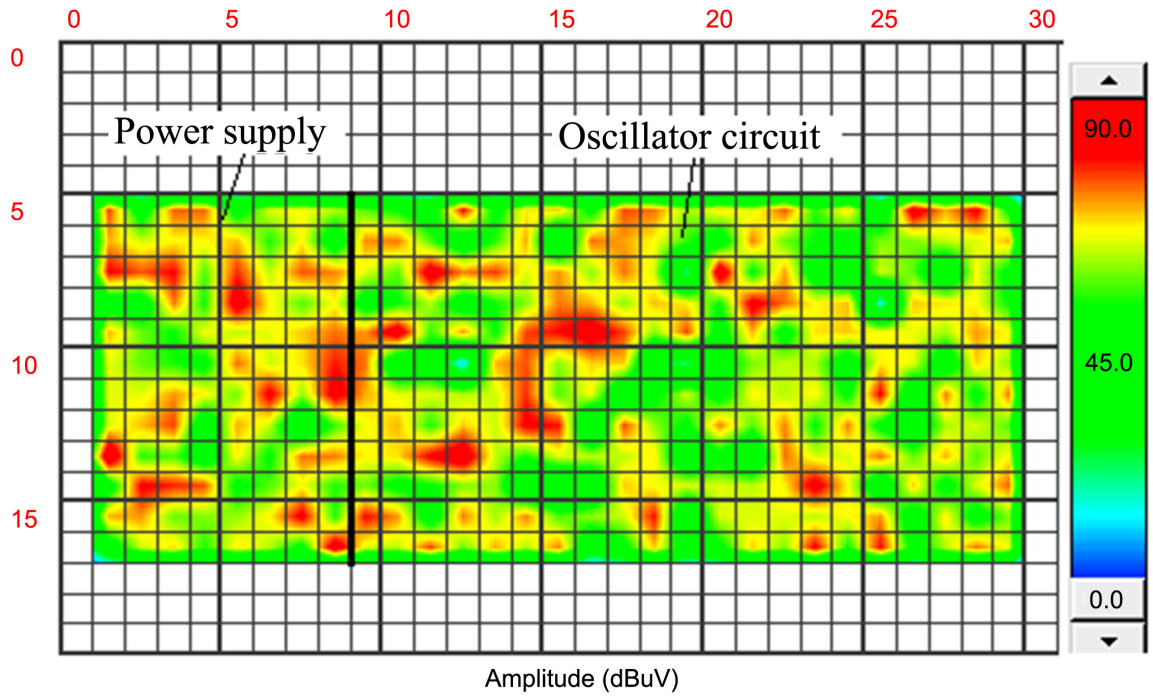

Figure 17. EF distribution in scanner plane in the case without EMC guarantee.

EF intensity is relatively hight and unhomogeneous depending on the circuit power of the individual electronic elements. At some positions, it rises to $90 \mathrm{dBuV}$.

However, the EF intensity reduces significantly when both units are metal sheilded and placed in distance of $10 \mathrm{~cm}$ one from other, i.e. is under the EMC guarantee (Figure 18).

From Figure 19 illustrated EF distribution in scanner plane, we see that the EF attenuation is about (25 - 30) dB in comparison to that without EMC guarantee.

It is clear that the electromagnetic noises from radio-electronic units should not act one on other, means the EMC is guaranteed when they are shielded with high $\mathrm{SE}$ and placed in a suitable distance. 


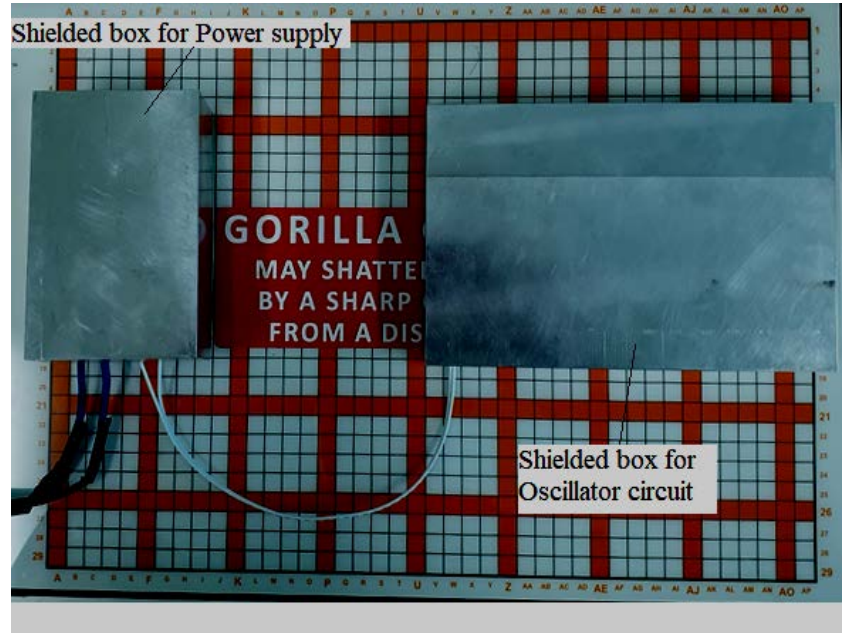

Figure 18. Two shielded circuits in diastance $10 \mathrm{~cm}$.

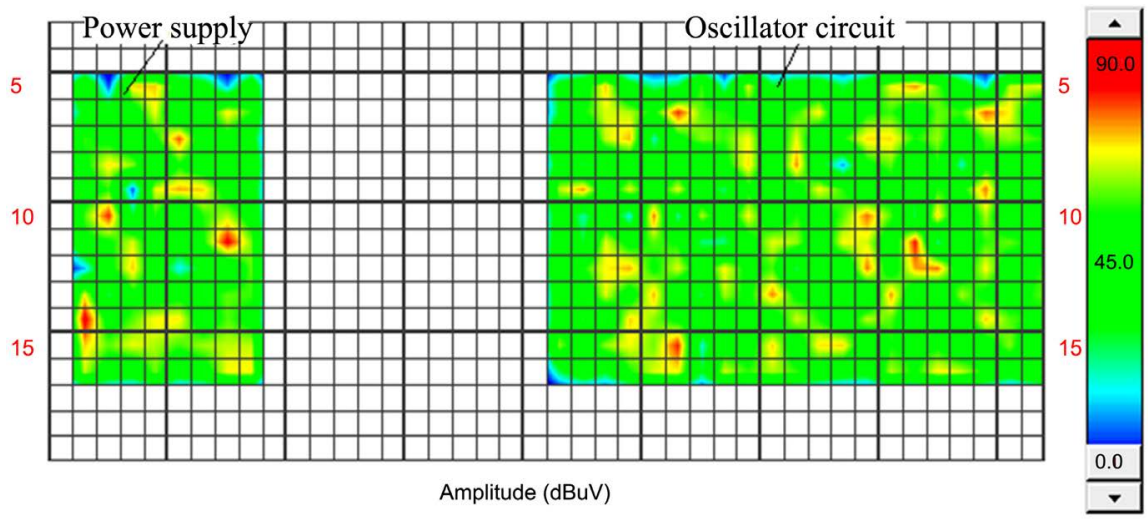

Figure 19. EF distribution in scanner plane in the case with EMC guarantee.

\section{Conclusions}

Based on Computer Simulation Technology, EMxpertEHX equipment, Inverse Distance Method, the EMC is simulated and tested followed to CISPR-22 standard. The EMC guarantee is investigated by two methods, metal shielding, distance estimation and then combination of them. The EMC is also experimentally evaluated for power supply and oscillator circuit. Although both methods are not new, but combination of them enhances the effectiveness to guarantee EMC, moreover the obtained results give us clear image about EMC, which can be used to test the capability to guarantee EMC of REUs in detail.

The results give us a hint to combine simulation and testing tools to automatically evaluate EMC of the REUs with different mechanical configurations, electronic designs at different estimated distances in the future.

\section{Conflicts of Interest}

The authors declare no conflicts of interest regarding the publication of this paper. 


\section{References}

[1] Balanis, C.A. (2015) Antenna Theory: Analysis and Design. Wiley, New York.

[2] Xiong, W., Jiang, M., Zhu, M., Zhu, B. and Lu, J. (2018) Analysis of Electromagnetic Shielding of IC Package with Thin Absorbing Material Coating Inside in Two Different Configuration. 2018 IEEE International Symposium on Electromagnetic Compatibility and 2018 IEEE Asia-Pacific Symposium on Electromagnetic Compatibility, Singapore, 14-18 May 2018, 1216-1221. https://doi.org/10.1109/ISEMC.2018.8393982

[3] Hwang, J.T., Han, Y.B., Park, H.H., Nah, W.S. and Kim, S.Y. (2015) Radiated Electromagnetic Immunity Analysis of VCO IC Stripling Method. 2015 10th International Workshop on the Electromagnetic Compatibility of Integrated Circuits, Edinburgh, 10-13 November 2015, 147-151. https://doi.org/10.1109/EMCCompo.2015.7358347

[4] Chien, T.T., Tu, B.T.M. and Do, T.N. (2016) Improvement of Shielding for the Electromagnetic Compatibility. 2016 International Conference on Electronics, Information, and Communications, Da Nang, Vietnam, 27-30 January 2016, 1-4.

[5] Antipin, A.S., Frizen, V.E., Sarapulov, F.N. and Kondratev, M.A. (2017) Power and Energy Parameters of the Inductor with an Electromagnetic Shield. 2017 15th International Conference on Electrical Machines, Drives and Power Systems, Sofia, Bulgaria, 1-3 June 2017, 406-409. https://doi.org/10.1109/ELMA.2017.7955473

[6] International Electrotechnical Committee (2009) Information Technology Equipment Radio Disturbance Characteristics - Limits and Methods Measurement.

[7] Tai, N.T., Hoan, P.K. and Hùng, P.T. (2011) Electromagnetic Compatibility. Army Publshing House, Hanoi.

[8] EMC (2008) Electromagnetic Compatibility Design Guide. Tecknit.

[9] Morari, C. and Balan, I. (2015) Methods for Determining Shielding Effectiveness of Materials. Electrotehnica, Electronica, Automatica, 63, 56-64.

[10] Chien, T.T., Tu, B.T.M. and Do, T.N. (2016) Improvement of Shielding for Electromagnetic Compatibility. IEIE Transactions on Smart Processing \& Computing, 5, 63-69. https://doi.org/10.5573/IEIESPC.2016.5.1.63

[11] Huang, P., Liu, Z. and Qi, J. (2016) Simulation Analysis of Electromagnetic Shielding Effectiveness in Ventilation Window of Waveguide. Journal of Residuals Science \& Technology, 13, 142-149.

[12] https://www.digitalengineering247.com/company/cst-computer-simulation-technol ogy

[13] https://www.mdltechnologies.co.uk/products/emxpert/

[14] Hemming, H.L. (1992) Architectural Electromagnetic Shielding Handbook: A Design and Specification Guide. The Institute of Electrical and Electronics Engineers, Inc., New York.

[15] Paul, C.R. (2006) Introduction to Electromagnetic Compatibility. John Wiley \& Sonc, Inc., New York. 